Segev Naveh Tassa ${ }^{1}$, Shani Ben Zichri ${ }^{2}$, Shiran Lacham-Hartman ${ }^{1}$, Ofek Oren ${ }^{3}$, Zeev Slobodnik ${ }^{4,5}$, Ekaterina Eremenko ${ }^{4,5}$, Debra Toiber ${ }^{4,5}$, Raz Jelinek ${ }^{2}$, Niv Papo ${ }^{1 *}$

${ }^{1}$ Avram and Stella Goldstein-Goren Department of Biotechnology Engineering and the National Institute of Biotechnology in the Negev, Ben-Gurion University of the Negev, P.O. Box 653, Beer-Sheva 84105, Israel. ${ }^{2}$ Department of Chemistry, Ben-Gurion University of the Negev, P.O. Box 653, Beer-Sheva 84105, Israel; ${ }^{3}$ The Shraga Segal Department of Microbiology, Immunology and Genetics, Faculty of Health Sciences, Ben-Gurion University of the Negev, P.O. Box 653, Beer-Sheva 84105, Israel; ${ }^{4}$ Department of Life Sciences, Ben-Gurion University of the Negev, P.O. Box 653, Beer-Sheva 84105, Israel; ${ }^{5}$ The Zlotowski Center for Neuroscience, Ben-Gurion University of the Negev, Beer Sheva, 84105, Israel. 84105, Israel.

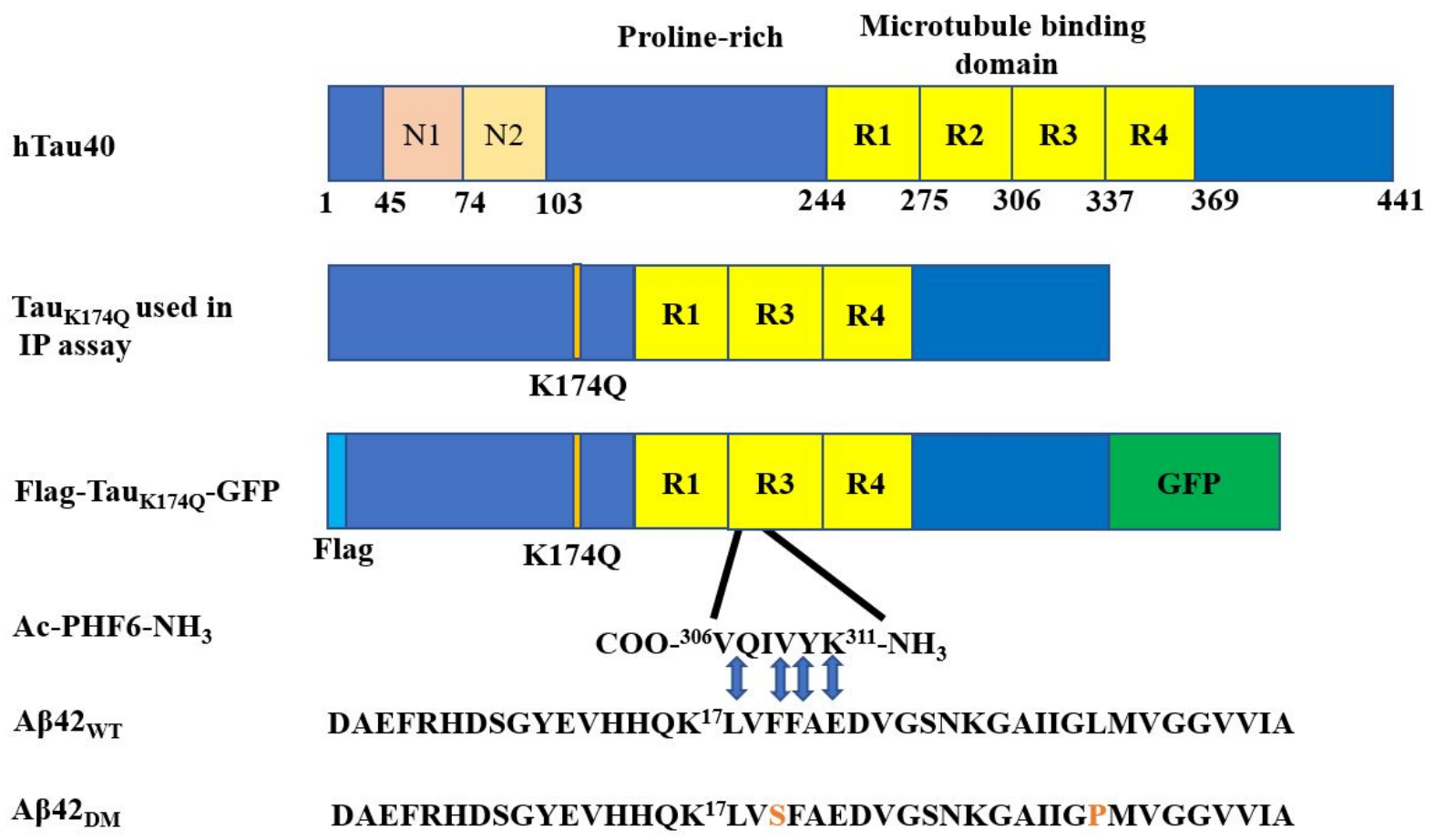

Figure S1. Scheme of tau, A $\beta 42$, and tau- and A $\beta 42$-derived fragments. Top to bottom, full length hTau40; ${ }^{1} \operatorname{tau}_{\mathrm{K} 174 \mathrm{Q}}$ used in IP assay, Flag-tau $\mathrm{K} 174 \mathrm{Q}_{\mathrm{Q}}$ GFP used in all other assays in which cells were transfected; PHF6 peptide sequence, $\mathrm{A} \beta 42_{\mathrm{WT}}$ and $\mathrm{A} \beta 42_{\mathrm{DM}}$ sequences are also shown. Blue arrows indicate hydrophobic interactions between $\mathrm{A} \beta 42_{\mathrm{WT}}$ and PHF6, as calculated by Miller et al. ${ }^{2}$ 


\section{A Mechanism for the Inhibition of tau Neurotoxicity: Studies with Artificial Membranes, Isolated Mitochondria and Intact Cells}

Segev Naveh Tassa ${ }^{1}$, Shani Ben Zichri ${ }^{2}$, Shiran Lacham-Hartman ${ }^{1}$, Ofek Oren ${ }^{3}$, Zeev Slobodnik ${ }^{4,5}$, Ekaterina Eremenko ${ }^{4,5}$, Debra Toiber ${ }^{4,5}$, Raz Jelinek ${ }^{2}$, Niv Papo ${ }^{1 *}$

${ }^{1}$ Avram and Stella Goldstein-Goren Department of Biotechnology Engineering and the National Institute of Biotechnology in the Negev, Ben-Gurion University of the Negev, P.O. Box 653, Beer-Sheva 84105, Israel. ${ }^{2}$ Department of Chemistry, Ben-Gurion University of the Negev, P.O. Box 653, Beer-Sheva 84105, Israel; ${ }^{3}$ The Shraga Segal Department of Microbiology, Immunology and Genetics, Faculty of Health Sciences, Ben-Gurion University of the Negev, P.O. Box 653, Beer-Sheva 84105, Israel; ${ }^{4}$ Department of Life Sciences, Ben-Gurion University of the Negev, P.O. Box 653, Beer-Sheva 84105, Israel; ${ }^{5}$ The Zlotowski Center for Neuroscience, Ben-Gurion University of the Negev, Beer Sheva, 84105, Israel. 84105, Israel.

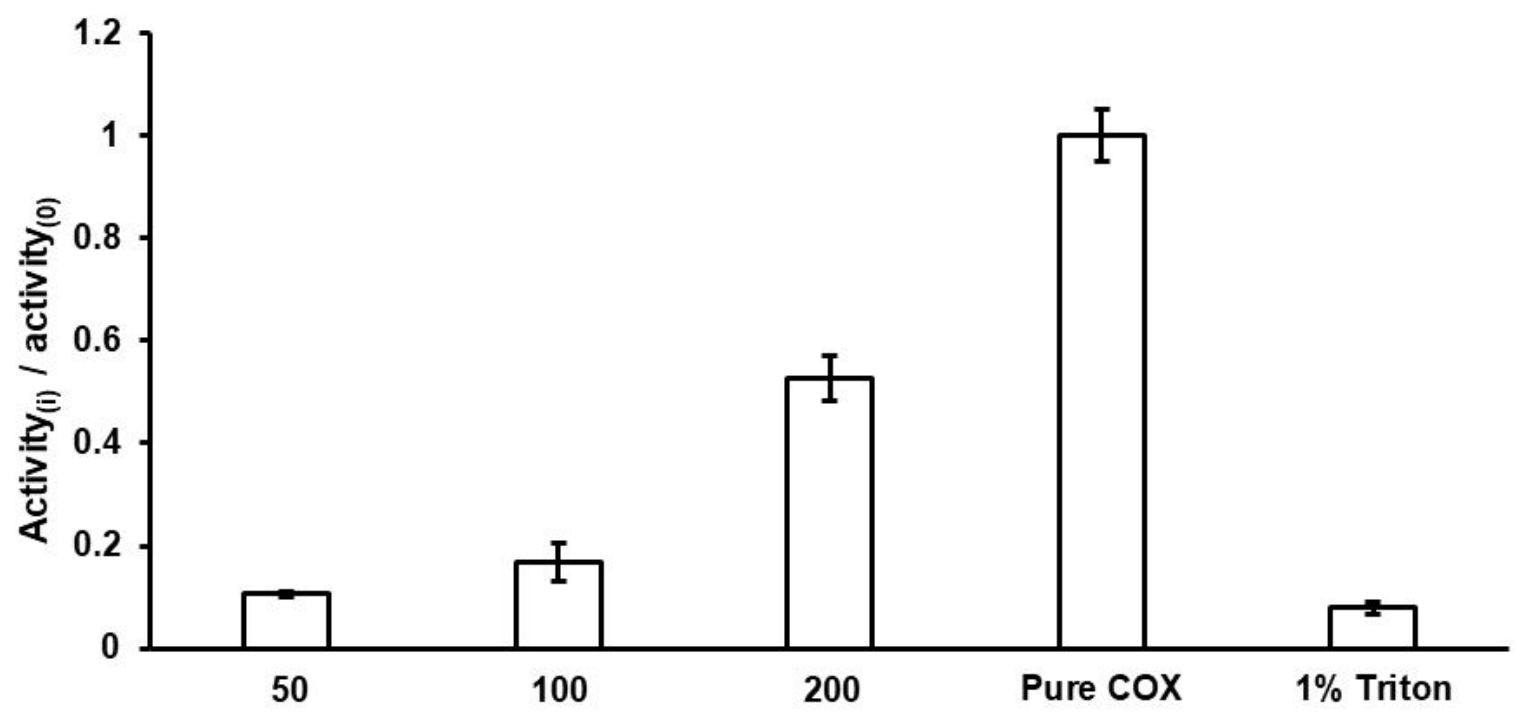

Figure S2: Cytochrome c oxidase (COX) activity. The activity of COX was measured using different concentrations of SMPs $(50-200 \mu \mathrm{g}$ total $\mathrm{protein} / \mathrm{mL})$. The values of activity $_{(\mathrm{i})} /$ activity $_{(0)}$ are the ratio of activities normalized to the positive control $\left(10 \mu \mathrm{g} / \mathrm{mL}\right.$ of pure COX). Activity ${ }_{(i)}$ indicates the COX activity of SMPs sample and Activity $_{(0)}$ is the COX activity of pure enzyme (positive control). For the negative control measurement, the COX activity was measured in the presence of Triton X-100 (4\% final concentration). Data are means $\pm \operatorname{SD}(n=3)$. 


\section{Supporting Information for \\ A Mechanism for the Inhibition of tau Neurotoxicity: Studies with Artificial Membranes, Isolated Mitochondria and Intact Cells}

Segev Naveh Tassa ${ }^{1}$, Shani Ben Zichri ${ }^{2}$, Shiran Lacham-Hartman ${ }^{1}$, Ofek Oren ${ }^{3}$, Zeev Slobodnik ${ }^{4,5}$, Ekaterina Eremenko ${ }^{4,5}$, Debra Toiber ${ }^{4,5}$, Raz Jelinek ${ }^{2}$, Niv Papo ${ }^{1 *}$

${ }^{1}$ Avram and Stella Goldstein-Goren Department of Biotechnology Engineering and the National Institute of Biotechnology in the Negev, Ben-Gurion University of the Negev, P.O. Box 653, Beer-Sheva 84105, Israel. ${ }^{2}$ Department of Chemistry, Ben-Gurion University of the Negev, P.O. Box 653, Beer-Sheva 84105, Israel; ${ }^{3}$ The Shraga Segal Department of Microbiology, Immunology and Genetics, Faculty of Health Sciences, Ben-Gurion University of the Negev, P.O. Box 653, Beer-Sheva 84105, Israel; ${ }^{4}$ Department of Life Sciences, Ben-Gurion University of the Negev, P.O. Box 653, Beer-Sheva 84105, Israel; ${ }^{5}$ The Zlotowski Center for Neuroscience, Ben-Gurion University of the Negev, Beer Sheva, 84105, Israel. 84105, Israel.

(A)

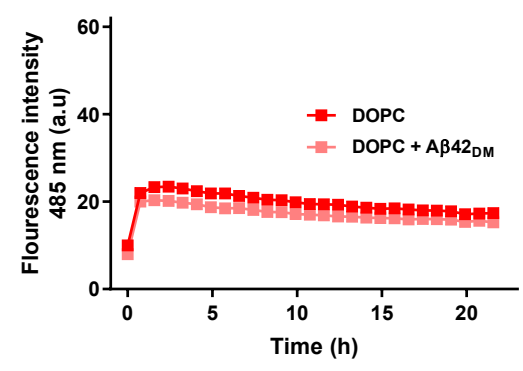

(B)

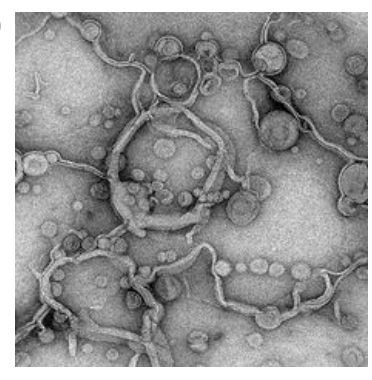

(C)

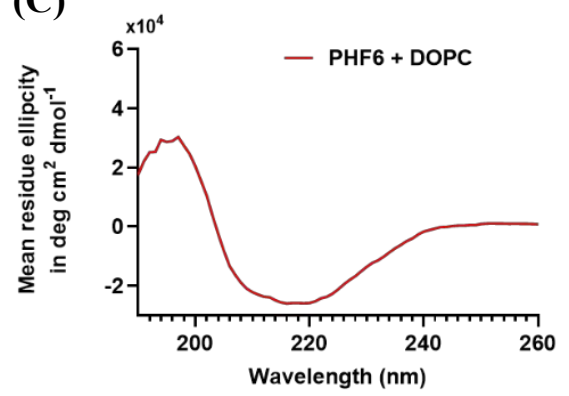

Figure S3: (A) ThT fluorescence was monitored over time upon co-incubation at $25{ }^{\circ} \mathrm{C}$ of PHF6 $(50 \mu \mathrm{M})$ with $\mathrm{A} \beta 42_{\mathrm{DM}}(175 \mathrm{nM})$ in the presence of DOPC. (B) TEM images taken $24 \mathrm{~h}$ post incubation at $25^{\circ} \mathrm{C}$ of PHF6 in DOPC. (C) Circular dichroism (CD) spectrum of synthetic PHF6 (50 $\mu \mathrm{M})$ in DOPC vesicles. CD spectra were obtained at wavelength of $185-260 \mathrm{~nm}$ using a quartz cuvette with a path length of $1 \mathrm{~mm}$. 


\section{Supporting Information for \\ A Mechanism for the Inhibition of tau Neurotoxicity: Studies with Artificial Membranes, Isolated Mitochondria and Intact Cells}

Segev Naveh Tassa ${ }^{1}$, Shani Ben Zichri ${ }^{2}$, Shiran Lacham-Hartman ${ }^{1}$, Ofek Oren ${ }^{3}$, Zeev Slobodnik ${ }^{4,5}$, Ekaterina Eremenko ${ }^{4,5}$, Debra Toiber ${ }^{4,5}$, Raz Jelinek ${ }^{2}$, Niv Papo ${ }^{1 *}$

${ }^{1}$ Avram and Stella Goldstein-Goren Department of Biotechnology Engineering and the National Institute of Biotechnology in the Negev, Ben-Gurion University of the Negev, P.O. Box 653, Beer-Sheva 84105, Israel. ${ }^{2}$ Department of Chemistry, Ben-Gurion University of the Negev, P.O. Box 653, Beer-Sheva 84105, Israel; ${ }^{3}$ The Shraga Segal Department of Microbiology, Immunology and Genetics, Faculty of Health Sciences, Ben-Gurion University of the Negev, P.O. Box 653, Beer-Sheva 84105, Israel; ${ }^{4}$ Department of Life Sciences, Ben-Gurion University of the Negev, P.O. Box 653, Beer-Sheva 84105, Israel; ${ }^{5}$ The Zlotowski Center for Neuroscience, Ben-Gurion University of the Negev, Beer Sheva, 84105, Israel. 84105, Israel.

\section{References}

(1) Fitzpatrick, A. W. P., Falcon, B., He, S., Murzin, A. G., Murshudov, G., Garringer, H. J., Crowther, R. A., Ghetti, B., Goedert, M., Scheres, S. H. W. Cryo-EM Structures of Tau Filaments from Alzheimer's Disease. Nature 2017, 547 (7662), 185-190.

(2) Miller, Y., Ma, B., Nussinov, R. Synergistic Interactions between Repeats in Tau Protein and A $\beta$ Amyloids May Be Responsible for Accelerated Aggregation via Polymorphic States. Biochemistry 2011, 50 (23), 5172-5181. 